\title{
Inferences under a Class of Finite Mixture Distributions Based on Generalized Order Statistics
}

\author{
Abd EL-Baset A. Ahmad ${ }^{1}$, Areej M. AL-Zaydi ${ }^{2}$ \\ ${ }^{1}$ Department of Mathematics, Assiut University, Assiut, Egypt \\ ${ }^{2}$ Department of Mathematics, Taif University, Taif, KSA \\ Email: abahmad2002@yahoo.com,aree.m.z@hotmail.com
}

Received May 1, 2012; revised January 2, 2013; accepted January 19, 2013

Copyright (C) 2013 Abd EL-Baset A. Ahmad, Areej M. AL-Zaydi. This is an open access article distributed under the Creative Commons Attribution License, which permits unrestricted use, distribution, and reproduction in any medium, provided the original work is properly cited.

\begin{abstract}
The main purpose of this paper is to obtain estimates of parameters, reliability and hazard rate functions of a heterogeneous population represented by finite mixture of two general components. The doubly Type II censoring of generalized order statistics scheme is used. Maximum likelihood and Bayes methods of estimation are used for this purpose. The two methods of estimation are compared via a Monte Carlo Simulation study.
\end{abstract}

Keywords: Generalized Order Statistics; Bayes Estimation; Heterogeneous Population; Monte Carlo Integration; Monte Carlo Simulation

\section{Introduction}

Let the random variable (rv) $T$ follow a class including some known lifetime models, its cumulative distribution function $(\mathrm{CDF})$ is given by

$$
F(t)=1-\exp [-\theta \lambda(t)], t>0,(\theta>0),
$$

and its probability density function (PDF) is given by

$$
f(t)=\theta \lambda^{\prime}(t) \exp [-\theta \lambda(t)], t>0,(\theta>0),
$$

where $\lambda^{\prime}(t)$ is the derivative of $\lambda(t)$ with respect to $t$ and $\lambda(t) \equiv \lambda(t ; \alpha)$ is a nonnegative continuous function of $t$ and $\alpha$ may be a vector of parameters, such that $\lambda(t) \rightarrow 0$ as $t \rightarrow 0^{+}$and $\lambda(t) \rightarrow \infty$ as $t \rightarrow \infty$.

The reliability function (RF) and hazard rate function (HRF) are given, respectively, by

$$
\begin{gathered}
R(t)=\exp [-\theta \lambda(t)], \\
H(t)=\theta \lambda^{\prime}(t),
\end{gathered}
$$

where

$$
H(.)=f(.) / R(.)
$$

Bayesian inferences based on finite mixture distribution have been discussed by several authors. Bayesian estimation of the mixing parameter, mean and reliability function of a mixture of two exponential lifetime distributions based on right censored samples considered by [1,2] es- timated the survival and hazard functions of a finite mixture of two Gompertz components by using type I and type II censored samples, using the maximum likelihood (ML) and Bayes methods. Based on type I censored samples from a finite mixture of two truncated type I generalized logistic components, [3] computed the Bayes estimates of parameters, reliability and hazard rate functions. [4] considered estimation for the mixed exponential distribution based on record statistics. [5] considered Bayes inference under a finite mixture of two compound Gompertz components model. [6] studied some properties of the mixture of two inverse Weibull distributions and obtained the estimates of the unknown parameters via the EM Algorithm.

[7] introduced the generalized order statistics (gos's). Ordinary order statistics, ordinary record values and sequential order statistics are, among others, special cases of gos's. The gos's have been considered extensively by many authors, among others, they are [8-20].

Mixtures of distributions arise frequently in life testing, reliability, biological and physical sciences. Some of the most important references that discussed different types of mixtures of distributions are a monograph by [21-23].

The PDF, CDF, RF and HRF of a finite mixture of two components of the class under study are given, respectively,

$$
f(t)=p_{1} f_{1}(t)+p_{2} f_{2}(t)
$$




$$
\begin{gathered}
F(t)=p_{1} F_{1}(t)+p_{2} F_{2}(t), \\
R(t)=p_{1} R_{1}(t)+p_{2} R_{2}(t), \\
H(t)=f(t) / R(t),
\end{gathered}
$$

where, for $j=1,2$, the mixing proportions $p_{j}$ are such that $0 \leq p_{j} \leq 1, p_{1}+p_{2}=1$ and $f_{j}(t), F_{j}(t), R_{j}(t)$ are given from (1), (2), (3) after using $\theta_{j}$ and $\lambda_{j}(t)$ instead of $\theta$ and $\lambda(t)$.

The property of identifiability is an important consideration on estimating the parameters in a mixture of distributions. Also, testing hypothesis, classification of random variables, can be meaning fully discussed only if the class of all finite mixtures is identifiable. Idenifiability of mixtures has been discussed by several authors, including [24-26].

Our aim of this paper is the estimation of the parameters and functions of these parameters of a class of finite mixture distributions based on doubly Type II censoring gos's using ML and Bayes methods. Illustrative example of Gompertz distribution is given and compared with the results obtained by previous researchers.

\section{Maximum Likelihood Estimation}

Let

$$
T_{s ; n, m, k}, T_{s+1 ; n, m, k}, \cdots, T_{r ; n, m, k}, 1 \leq s<r \leq n, k>0,
$$

be the $(r-s)$ gos's drawn from a mixture of two components of the class (2). Based on this doubly censored sample, the likelihood function can be written [27] as

$$
L(\theta \mid t)=\left\{\begin{array}{rr}
c_{1}\left\{\prod_{i=s}^{r}\left[R\left(t_{i}\right)\right]^{m} f\left(t_{i}\right)\right\}\left[R\left(t_{r}\right)\right]^{\gamma_{r+1}} & \\
\quad \times \sum_{\ell=0}^{s-1} \omega_{\ell}^{(s)}\left[R\left(t_{s}\right)\right]^{(s-\ell-1)(m+1)}, & m \neq-1, \\
c_{2}\left[R\left(t_{r}\right)\right]^{k}\left[\ln R\left(t_{s}\right)\right]^{s-1} \prod_{i=s}^{r} H\left(t_{i}\right), & m=-1,
\end{array}\right.
$$

where $t=\left(t_{s}, \cdots, t_{r}\right), \quad \theta \in \Theta, \Theta$ is the parameter space, and

$$
\left.\begin{array}{l}
c_{1}=\frac{(-1)^{s-1} C_{r-1}}{(m+1)^{s-1}(s-1) !}, c_{2}=\frac{(-1)^{s-1} k^{r}}{(s-1) !}, \\
C_{r-1}=\prod_{j=1}^{r} \gamma_{j}, \gamma_{r}=k+(n-r)(m+1), \\
\omega_{\ell}^{(s)}=(-1)^{\ell}\left(\begin{array}{c}
s-1 \\
\ell
\end{array}\right) .
\end{array}\right\}
$$

For definition and various distributional properties of gos's, see [7, 28].

The likelihood function (9) and maximum likelihood estimates (MLE's) can be obtained by using (1) and (5) in two cases, regarding to $m$ value, as follows.

\subsection{MLE's When $m \neq-1$}

In this case, substituting (1), (5) in (9), the likelihood function takes the form

$$
\begin{aligned}
L(\theta \mid \boldsymbol{t})= & c_{1}\left\{\prod_{i=s}^{r}\left[p_{1} R_{1}\left(t_{i}\right)+p_{2} R_{2}\left(t_{i}\right)\right]^{m}\right. \\
\left.\times\left[p_{1} f_{1}\left(t_{i}\right)+p_{2} f_{2}\left(t_{i}\right)\right]\right\} & \\
& \times\left[p_{1} R_{1}\left(t_{r}\right)+p_{2} R_{2}\left(t_{r}\right)\right]^{\gamma_{r+1}} \\
& \times \sum_{\ell=0}^{s-1} \omega_{\ell}^{(s)}\left[p_{1} R_{1}\left(t_{s}\right)+p_{2} R_{2}\left(t_{s}\right)\right]^{(s-\ell-1)(m+1)} .
\end{aligned}
$$

Take the logarithm of (10), we have

$$
\begin{aligned}
& \ell(\theta) \equiv \ln L(\theta \mid \boldsymbol{t})=\ln c_{1}+m \sum_{i=s}^{r} \ln \left[p_{1} R_{1}\left(t_{i}\right)+p_{2} R_{2}\left(t_{i}\right)\right] \\
& +\sum_{i=s}^{r} \ln \left[p_{1} f_{1}\left(t_{i}\right)+p_{2} f_{2}\left(t_{i}\right)\right]+\gamma_{r+1} \ln \left[p_{1} R_{1}\left(t_{r}\right)+p_{2} R_{2}\left(t_{r}\right)\right] \\
& +\ln \left\{\sum_{\ell=0}^{s-1} \omega_{\ell}^{(s)}\left[p_{1} R_{1}\left(t_{s}\right)+p_{2} R_{2}\left(t_{s}\right)\right]^{(s-\ell-1)(m+1)}\right\},
\end{aligned}
$$

where $p_{1}=p, \quad p_{2}=1-p$.

Differentiating (11) with respect to the parameters $p, \theta_{j}$ and $\alpha_{j}$ (involved in $\lambda$ ) and equating to zero gives the following likelihood equations

$$
\frac{\partial \ell}{\partial p}=m \sum_{i=s}^{r} \vartheta^{*}\left(t_{i}\right)+\sum_{i=s}^{r} \vartheta\left(t_{i}\right)+\gamma_{r+1} \vartheta^{*}\left(t_{r}\right) \frac{\sum_{\ell=0}^{s-1} \delta \tau_{\ell}\left(t_{s}\right) \vartheta^{*}\left(t_{s}\right)}{\sum_{\ell=0}^{s-1} \tau_{\ell}\left(t_{s}\right)}
$$$$
=0 \text {, }
$$

$$
\begin{aligned}
\frac{\partial \ell}{\partial \theta_{j}}= & -m \sum_{i=s}^{r} p_{j} \lambda_{j}\left(t_{i}\right) \psi_{j}^{*}\left(t_{i}\right)+\sum_{i=s}^{r} p_{j} \xi_{j}\left(t_{i}\right) \psi_{j}\left(t_{i}\right) \\
& -\gamma_{r+1} p_{j} \lambda_{j}\left(t_{r}\right) \psi_{j}^{*}\left(t_{r}\right) \\
& -\frac{\sum_{\ell=0}^{s-1} \delta \tau_{\ell}\left(t_{s}\right) p_{j} \lambda_{j}\left(t_{s}\right) \psi_{j}^{*}\left(t_{s}\right)}{\sum_{\ell=0}^{s-1} \tau_{\ell}\left(t_{s}\right)}=0, \quad j=1,2,
\end{aligned}
$$

$$
\begin{aligned}
\frac{\partial \ell}{\partial \alpha_{j}}= & -m \sum_{i=s}^{r} p_{j} \theta_{j} \frac{\partial \lambda_{j}\left(t_{i}\right)}{\partial \alpha_{j}} \psi_{j}^{*}\left(t_{i}\right)+\sum_{i=s}^{r} p_{j} \xi_{j}^{*}\left(t_{i}\right) \psi_{j}\left(t_{i}\right) \\
& -\gamma_{r+1} p_{j} \theta_{j} \frac{\partial \lambda_{j}\left(t_{r}\right)}{\partial \alpha_{j}} \psi_{j}^{*}\left(t_{r}\right) \\
& -\sum_{\ell=0}^{s-1} \delta \tau_{\ell}\left(t_{s}\right) p_{j} \theta_{j} \frac{\partial \lambda_{j}\left(t_{s}\right)}{\partial \alpha_{j}} \psi_{j}^{*}\left(t_{s}\right) \\
\sum_{\ell=0}^{s-1} \tau_{\ell}\left(t_{s}\right) & =0, \quad j=1,2
\end{aligned}
$$

where, for $j=1,2$ 


$$
\left.\begin{array}{l}
\xi_{j}\left(t_{i}\right)=\left[\frac{1}{\theta_{j}}-\lambda_{j}\left(t_{i}\right)\right], \xi_{j}^{*}\left(t_{i}\right)=\left[\frac{1}{\lambda_{j}^{\prime}\left(t_{i}\right)} \frac{\partial \lambda_{j}^{\prime}\left(t_{i}\right)}{\partial \alpha_{j}}-\theta_{j} \frac{\partial \lambda_{j}\left(t_{i}\right)}{\partial \alpha_{j}}\right], \vartheta\left(t_{i}\right)=\frac{f_{1}\left(t_{i}\right)-f_{2}\left(t_{i}\right)}{f\left(t_{i}\right)}, \\
\vartheta^{*}\left(t_{i}\right)=\frac{R_{1}\left(t_{i}\right)-R_{2}\left(t_{i}\right)}{R\left(t_{i}\right)}, \psi_{j}\left(t_{i}\right)=\frac{f_{j}\left(t_{i}\right)}{f\left(t_{i}\right)}, \quad \psi_{j}^{*}\left(t_{i}\right)=\frac{R_{j}\left(t_{i}\right)}{R\left(t_{i}\right)}, \tau_{\ell}\left(t_{s}\right)=\omega_{\ell}^{(s)} R\left(t_{s}\right)^{\delta}, \delta=(s-\ell-1)(m+1)
\end{array}\right\}
$$

The solution of the five nonlinear likelihood Equations (12) using numerical method, yields the MLE's $\hat{p}, \hat{\theta}_{1}, \hat{\theta}_{2}, \hat{\alpha}_{1}$ and $\hat{\alpha}_{2}$.

\subsection{MLE's When $m=-1$}

The likelihood function takes the form

$$
L(\theta \mid \boldsymbol{t})=c_{2}\left[p_{1} R_{1}\left(t_{r}\right)+p_{2} R_{2}\left(t_{r}\right)\right]^{k} \times\left(\ln \left[p_{1} R_{1}\left(t_{s}\right)+p_{2} R_{2}\left(t_{s}\right)\right]\right)^{s-1} \times \prod_{i=s}^{r} \frac{\left[p_{1} f_{1}\left(t_{i}\right)+p_{2} f_{2}\left(t_{i}\right)\right]}{\left[p_{1} R_{1}\left(t_{i}\right)+p_{2} R_{2}\left(t_{i}\right)\right]} .
$$

So, from (14)

$$
\begin{aligned}
& \ell(\theta) \propto k \ln \left[p_{1} R_{1}\left(t_{r}\right)+p_{2} R_{2}\left(t_{r}\right)\right]+(s-1) \ln \left(\ln \left[p_{1} R_{1}\left(t_{s}\right)+p_{2} R_{2}\left(t_{s}\right)\right]\right) \\
&+\sum_{i=s}^{r}\left(\ln \left[p_{1} f_{1}\left(t_{i}\right)+p_{2} f_{2}\left(t_{i}\right)\right]-\ln \left[p_{1} R_{1}\left(t_{i}\right)+p_{2} R_{2}\left(t_{i}\right)\right]\right) .
\end{aligned}
$$

Differentiating (15) with respect to the parameters $p, \theta_{j}$ and $\alpha_{j}$ and equating to zero gives the following likelihood equations

$$
\left.\begin{array}{rl}
\frac{\partial \ell}{\partial p} & =k \vartheta^{*}\left(t_{r}\right)+(s-1) \vartheta^{*}\left(t_{s}\right) \varphi\left(t_{s}\right)+\sum_{i=s}^{r}\left(\vartheta\left(t_{i}\right)-\vartheta^{*}\left(t_{i}\right)\right)=0, \\
\frac{\partial \ell}{\partial \theta_{j}} & =-k p_{j} \lambda_{j}\left(t_{r}\right) \psi_{j}^{*}\left(t_{r}\right)-(s-1) p_{j} \lambda_{j}\left(t_{s}\right) \psi_{j}^{*}\left(t_{s}\right) \varphi\left(t_{s}\right)+\sum_{i=s}^{r}\left(p_{j} \xi_{j}\left(t_{i}\right) \psi_{j}\left(t_{i}\right)+p_{j} \lambda_{j}\left(t_{i}\right) \psi_{j}^{*}\left(t_{i}\right)\right) \\
& =0, \quad j=1,2, \\
\frac{\partial \ell}{\partial \alpha_{j}} & =-k p_{j} \theta_{j} \frac{\partial \lambda_{j}\left(t_{r}\right)}{\partial \alpha_{j}} \psi_{j}^{*}\left(t_{r}\right)-(s-1) p_{j} \theta_{j} \frac{\partial \lambda_{j}\left(t_{s}\right)}{\partial \alpha_{j}} \psi_{j}^{*}\left(t_{s}\right) \varphi\left(t_{s}\right)+\sum_{i=s}^{r}\left(p_{j} \xi_{j}^{*}\left(t_{i}\right) \psi_{j}\left(t_{i}\right)+p_{j} \theta_{j} \frac{\partial \lambda_{j}\left(t_{i}\right)}{\partial \alpha_{j}} \psi_{j}^{*}\left(t_{i}\right)\right) \\
& =0, \quad j=1,2
\end{array}\right\}
$$

where

$$
\varphi\left(t_{i}\right)=\frac{1}{\ln R\left(t_{i}\right)} .
$$

The solution of the five nonlinear likelihood Equations (16) using numerical method, yields the MLE's $\hat{p}, \hat{\theta}_{1}, \hat{\theta}_{2}, \hat{\alpha}_{1}$ and $\hat{\alpha}_{2}$.

\section{Bayes Estimation}

In this section, Bayesian estimation for the parameters of a class of finite mixture distributions is considered under squared error and Linex (Linear-Exponential) loss functions.

We shall use the conjugate prior density, that was suggested by [29], in the following form

$$
\begin{aligned}
& \pi(\theta ; v) \propto C(\theta ; v) \exp [-D(\theta ; v)], \\
& \theta=\left(p, \theta_{1}, \theta_{2}, \alpha_{1}, \alpha_{2}\right), v \in \Omega,
\end{aligned}
$$

where $\Omega$ is the hyperparameter space.

\subsection{Bayes Estimates When $m \neq-1$}

It follows, from (10) and (18), that the posterior density function is given by

$$
\begin{aligned}
& \pi^{*}(\theta \mid \boldsymbol{t})=A_{1} C(\theta ; v) \exp [-D(\theta ; v)] \\
& \times\left\{\prod_{i=s}^{r}\left[p_{1} R_{1}\left(t_{i}\right)+p_{2} R_{2}\left(t_{i}\right)\right]^{m}\left[p_{1} f_{1}\left(t_{i}\right)+p_{2} f_{2}\left(t_{i}\right)\right]\right\} \\
& \times\left[p_{1} R_{1}\left(t_{r}\right)+p_{2} R_{2}\left(t_{r}\right)\right]^{\gamma_{r+1}} \\
& \times \sum_{\ell=0}^{s-1} \omega_{\ell}^{(s)}\left[p_{1} R_{1}\left(t_{s}\right)+p_{2} R_{2}\left(t_{s}\right)\right]^{\delta}
\end{aligned}
$$

where

$$
A_{1}^{-1}=\int_{\theta} \pi(\theta ; v) L(\theta \mid \boldsymbol{t}) \mathrm{d} \theta .
$$

The Bayes estimator of a function, say $u(\theta)$, under 
squared error and Linex loss functions is given, respectively, by

$$
\begin{aligned}
\hat{u}_{B S}= & E(u(\theta) \mid \boldsymbol{t})=\int_{\theta} u(\theta) \pi^{*}(\theta \mid \boldsymbol{t}) \mathrm{d} \theta, \\
\hat{u}_{B L} & =\frac{1}{a} \ln \left[E\left(\mathrm{e}^{-a u(\theta)} \mid \boldsymbol{t}\right)\right] \\
& =-\frac{1}{a} \ln \left[\int_{\theta} \mathrm{e}^{-a u(\theta)} \pi^{*}(\theta \mid \boldsymbol{t}) \mathrm{d} \theta\right],
\end{aligned}
$$

where the integral is taken over the five dimensional space and $a \neq 0$.

To compute the integral, we can use the Monte Carlo Integration (MCI) method in the form

$$
\begin{gathered}
\hat{u}_{B S}=\frac{\sum_{j=1}^{M} u\left(\theta^{j}\right) L\left(\theta^{j} \mid \boldsymbol{t}\right)}{\sum_{j=1}^{M} L\left(\theta^{j} \mid \boldsymbol{t}\right)}, \\
\hat{u}_{B L}=-\frac{1}{a} \ln \left[\frac{\sum_{j=1}^{M} \mathrm{e}^{-a u\left(\theta^{j}\right)} L\left(\theta^{j} \mid \boldsymbol{t}\right)}{\sum_{j=1}^{M} L\left(\theta^{j} \mid \boldsymbol{t}\right)}\right],
\end{gathered}
$$

where $\theta^{j}, j=1,2, \cdots, M$ is generated from the PDF $\pi(\theta ; v)$, for more details see [30].

Under squared error and Linex loss functions, we can obtain the Bayes estimator of the parameter $p$, by generating

$$
\left(p^{j}, \theta_{1}^{j}, \theta_{2}^{j}, \alpha_{1}^{j}, \alpha_{2}^{j}\right) j=1,2, \cdots, M
$$

from the prior (18) and setting $u(\theta)=p$ in (23) and (24). The Bayes estimates of $\theta_{1}, \theta_{2}, \alpha_{1}$ and $\alpha_{2}$ can be similarly computed.

\subsection{Bayes Estimates When $m=-1$}

The posterior density function can be obtained from (14) and (18), as

$$
\begin{aligned}
\pi^{*}(\theta \mid \boldsymbol{t})= & A_{2} C(\theta ; v) \exp [-D(\theta ; v)] \\
& \times\left[p_{1} R_{1}\left(t_{r}\right)+p_{2} R_{2}\left(t_{r}\right)\right]^{k} \\
& \times\left(\ln \left[p_{1} R_{1}\left(t_{s}\right)+p_{2} R_{2}\left(t_{s}\right)\right]\right)^{s-1} \\
& \times \prod_{i=s}^{r} \frac{\left[p_{1} f_{1}\left(t_{i}\right)+p_{2} f_{2}\left(t_{i}\right)\right]}{\left[p_{1} R_{1}\left(t_{i}\right)+p_{2} R_{2}\left(t_{i}\right)\right]}
\end{aligned}
$$

where

$$
A_{2}^{-1}=\int_{\theta} \pi(\theta ; v) L(\theta \mid \boldsymbol{t}) \mathrm{d} \theta .
$$

Under squared error and Linex loss functions, we can obtain the Bayes estimator of the parameter $p$, by generating

$$
\left(p^{j}, \theta_{1}^{j}, \theta_{2}^{j}, \alpha_{1}^{j}, \alpha_{2}^{j}\right) j=1,2, \cdots, M
$$

from the prior (18) and setting $u(\theta)=p$ in (23) and (24). The Bayes estimates of $\theta_{1}, \theta_{2}, \alpha_{1}$ and $\alpha_{2}$ can be similarly computed.

\section{Example}

\subsection{Gompertz Components}

\subsubsection{Maximum Likelihood Estimation}

Suppose that, for $j=1,2$ and $t>0, \theta_{j}=1$,

$$
\lambda_{j}(t)=\frac{1}{\alpha_{j}}\left[\mathrm{e}^{\alpha_{j} t}-1\right]
$$

so

$$
\dot{\lambda}_{j}(t)=\mathrm{e}^{\alpha_{j} t} .
$$

In this case, the $j^{\text {th }}$ subpopulation is Gompertz distribution with parameter $\alpha_{j}>0$.

For $m \neq-1$ by substituting $\lambda_{j}(t)$ and $\dot{\lambda}_{j}(t)$ in (12), we have the following nonlinear equations

$$
\begin{aligned}
& \frac{\partial \ell}{\partial p}=m \sum_{i=s}^{r} \vartheta^{*}\left(t_{i}\right) \\
& +\sum_{i=s}^{r} \vartheta\left(t_{i}\right)+\gamma_{r+1} \vartheta^{*}\left(t_{r}\right) \\
& +\frac{\sum_{\ell=0}^{s-1} \delta \tau_{\ell}\left(t_{s}\right) \vartheta^{*}\left(t_{s}\right)}{\sum_{\ell=0}^{s-1} \tau_{\ell}\left(t_{s}\right)}=0, \\
& \frac{\partial \ell}{\partial \alpha_{j}}=-m \sum_{i=s}^{r} p_{j} \varpi_{j}\left(t_{i}\right) \psi_{j}^{*}\left(t_{i}\right) \\
& +\sum_{i=s}^{r} p_{j} \xi_{j}^{*}\left(t_{i}\right) \psi_{j}\left(t_{i}\right) \\
& -\gamma_{r+1} p_{j} \varpi_{j}\left(t_{r}\right) \psi_{j}^{*}\left(t_{r}\right) \\
& -\frac{\sum_{\ell=0}^{s-1} \delta \tau_{\ell}\left(t_{s}\right) p_{j} \varpi_{j}\left(t_{s}\right) \psi_{j}^{*}\left(t_{s}\right)}{\sum_{\ell=0}^{s-1} \tau_{\ell}\left(t_{s}\right)}=0, \quad j=1,2
\end{aligned}
$$

where, for $j=1,2$

$$
\left.\begin{array}{l}
\xi_{j}\left(t_{i}\right)=1-\frac{1}{\alpha_{j}}\left(\mathrm{e}^{\alpha_{j} t}-1\right), \\
\xi_{j}^{*}\left(t_{i}\right)=t_{i}-\varpi_{j}\left(t_{i}\right), \\
\varpi_{j}\left(t_{i}\right)=\frac{1}{\alpha_{j}^{2}}\left[1+\left(\alpha_{j} t_{i}-1\right) \mathrm{e}^{\alpha_{j} t_{i}}\right]
\end{array}\right\},
$$

$\hat{p}, \hat{\alpha}_{1}$ and $\hat{\alpha}_{2}$ are the solution of the above nonlinear equations.

Also, for $m=-1$ substituting $\lambda_{j}(t)$ and $\dot{\lambda}_{j}(t)$ in (13), (16) and (17), we have the following nonlinear equations: 


$$
\left.\begin{array}{l}
\frac{\partial \ell}{\partial p}=k \vartheta^{*}\left(t_{r}\right)+(s-1) \vartheta^{*}\left(t_{s}\right) \varphi\left(t_{s}\right)+\sum_{i=s}^{r}\left(\vartheta\left(t_{i}\right)-\vartheta^{*}\left(t_{i}\right)\right)=0 \\
\left.\frac{\partial \ell}{\partial \alpha_{j}}=-k p_{j} \varpi_{j}\left(t_{r}\right) \psi_{j}^{*}\left(t_{r}\right)-(s-1) p_{j} \varpi_{j}\left(t_{s}\right) \psi_{j}^{*}\left(t_{s}\right) \varphi\left(t_{s}\right)+\sum_{i=s}^{r}\left(p_{j} \xi_{j}^{*}\left(t_{i}\right) \psi_{j}\left(t_{i}\right)+p_{j} \varpi_{j}\left(t_{i}\right) \psi_{j}^{*}\left(t_{i}\right)\right)=0, j=1,2\right\}
\end{array}\right\}
$$

$\hat{p}, \hat{\alpha}_{1}$ and $\hat{\alpha}_{2}$ are the solution of the above nonlinear equations.

\section{Special cases}

1) Upper order statistics
If we put $m=0$ and $k=1$ in (10),

$$
\left(\gamma_{r}=n-r+1\right)
$$

the likelihood function takes the form

$$
L(\theta \mid \boldsymbol{t})=c_{1} \prod_{i=s}^{r}\left[p_{1} f_{1}\left(t_{i}\right)+p_{2} f_{2}\left(t_{i}\right)\right]\left[p_{1} R_{1}\left(t_{r}\right)+p_{2} R_{2}\left(t_{r}\right)\right]^{n-r} \times \sum_{\ell=0}^{s-1} \omega_{\ell}^{(s)}\left[p_{1} R_{1}\left(t_{s}\right)+p_{2} R_{2}\left(t_{s}\right)\right]^{s-\ell-1} .
$$

Substituting $m=0, k=1$ and $\gamma_{r}=n-r+1$ in (27), we have the following nonlinear equations

$$
\left.\begin{array}{l}
\frac{\partial \ell}{\partial p}=\sum_{i=s}^{r} \vartheta\left(t_{i}\right)+(n-r) \vartheta^{*}\left(t_{r}\right)+\frac{\sum_{\ell=0}^{s-1} \delta \tau_{\ell}\left(t_{s}\right) \vartheta^{*}\left(t_{s}\right)}{\sum_{\ell=0}^{s-1} \tau_{\ell}\left(t_{s}\right)}=0, \\
\frac{\partial \ell}{\partial \alpha_{j}}=\sum_{i=s}^{r} p_{j} \xi_{j}^{*}\left(t_{i}\right) \psi_{j}\left(t_{i}\right)-(n-r) p_{j} \varpi_{j}\left(t_{r}\right) \psi_{j}^{*}\left(t_{r}\right) \frac{\sum_{\ell=0}^{s-1} \delta \tau_{\ell}\left(t_{s}\right) p_{j} \varpi_{j}\left(t_{s}\right) \psi_{j}^{*}\left(t_{s}\right)}{\sum_{\ell=0}^{s-1} \tau_{\ell}\left(t_{s}\right)}=0, \quad j=1,2
\end{array}\right\},
$$

where $\delta=s-\ell-1$.

The solution of the nonlinear likelihood equations (31) gives the MLE's $\hat{p}, \hat{\alpha}_{1}$ and $\hat{\alpha}_{2}$.

\section{2) Upper record values}

If we put $k=1$ in (14), $\left(\gamma_{r}=1\right)$ the likelihood function takes the form

$$
L(\theta \mid \boldsymbol{t})=c_{2}\left[p_{1} R_{1}\left(t_{r}\right)+p_{2} R_{2}\left(t_{r}\right)\right]\left(\ln \left[p_{1} R_{1}\left(t_{s}\right)+p_{2} R_{2}\left(t_{s}\right)\right]\right)^{s-1} \times \prod_{i=s}^{r} \frac{\left[p_{1} f_{1}\left(t_{i}\right)+p_{2} f_{2}\left(t_{i}\right)\right]}{\left[p_{1} R_{1}\left(t_{i}\right)+p_{2} R_{2}\left(t_{i}\right)\right]} .
$$

Substituting $k=1$ in (29), we have the following nonlinear equations

$$
\left.\begin{array}{l}
\frac{\partial \ell}{\partial p}=(s-1) \vartheta^{*}\left(t_{s}\right) \varphi\left(t_{s}\right)+\sum_{i=s}^{r} \vartheta\left(t_{i}\right)-\sum_{i=s}^{r-1} \vartheta^{*}\left(t_{i}\right)=0, \\
\frac{\partial \ell}{\partial \alpha_{j}}=\sum_{i=s}^{r} p_{j} \xi_{j}^{*}\left(t_{i}\right) \psi_{j}\left(t_{i}\right)-(s-1) p_{j} \varpi_{j}\left(t_{s}\right) \psi_{j}^{*}\left(t_{s}\right) \varphi\left(t_{s}\right)+\sum_{i=s}^{r-1} p_{j} \varpi_{j}\left(t_{i}\right) \psi_{j}^{*}\left(t_{i}\right)=0, j=1,2
\end{array}\right\},
$$

The solution of the nonlinear likelihood Equations (33) gives the MLE's $\hat{p}, \hat{\alpha}_{1}$ and $\hat{\alpha}_{2}$.

\subsubsection{Bayes Estimation}

Let $p, \alpha_{1}$ and $\alpha_{2}$ are independent random variables such that $p \sim \operatorname{Beta}\left(b_{1}, b_{2}\right)$ and for $j=1,2, \alpha_{j}$ to follow a left truncated exponential density with parameter $d_{j}\left(\operatorname{LTE}\left(d_{j}\right)\right)$, as used by [2]. A joint prior density function is then given by

$$
\pi(\theta ; v) \propto p_{1}^{b_{1}-1} p_{2}^{b_{2}-1} \exp \left[-\sum_{j=1}^{2} \frac{\left(\alpha_{j}-1\right)}{d_{j}}\right],
$$

$$
0<p_{1}<1, \alpha_{j}>1,\left(b_{1}, b_{2}, d_{1}, d_{2}\right)>0
$$

and $p_{2}=1-p_{1}$.

For $m \neq-1$, the posterior density function $\pi^{*}(\theta \mid \boldsymbol{t})$ then takes the form

$$
\begin{aligned}
& \pi^{*}(\theta \mid \boldsymbol{t}) \propto p_{1}^{b_{1}-1} p_{2}^{b_{2}-1} \exp \left[-\sum_{j=1}^{2} \frac{\left(\alpha_{j}-1\right)}{d_{j}}\right] \\
& \times\left\{\prod_{i=s}^{r}\left[p_{1} R_{1}\left(t_{i}\right)+p_{2} R_{2}\left(t_{i}\right)\right]^{m} \times\left[p_{1} f_{1}\left(t_{i}\right)+p_{2} f_{2}\left(t_{i}\right)\right]\right\} \\
& \times\left[p_{1} R_{1}\left(t_{r}\right)+p_{2} R_{2}\left(t_{r}\right)\right]^{\gamma_{r+1}} \sum_{\ell=0}^{s-1} \omega_{\ell}^{(s)}\left[p_{1} R_{1}\left(t_{s}\right)+p_{2} R_{2}\left(t_{s}\right)\right]^{\delta}
\end{aligned}
$$

where 
For $\mathrm{m}=-1$ the posterior density function $\pi^{*}(\theta \mid \boldsymbol{t})$ then takes the form

$$
\pi^{*}(\theta \mid \boldsymbol{t}) \propto p_{1}^{b_{1}-1} p_{2}^{b_{2}-1} \exp \left[-\sum_{j=1}^{2} \frac{\left(\alpha_{j}-1\right)}{d_{j}}\right]\left[p_{1} R_{1}\left(t_{r}\right)+p_{2} R_{2}\left(t_{r}\right)\right]^{k} \times\left(\ln \left[p_{1} R_{1}\left(t_{s}\right)+p_{2} R_{2}\left(t_{s}\right)\right]\right)^{s-1} \prod_{i=s}^{r} \frac{\left[p_{1} f_{1}\left(t_{i}\right)+p_{2} f_{2}\left(t_{i}\right)\right]}{\left[p_{1} R_{1}\left(t_{i}\right)+p_{2} R_{2}\left(t_{i}\right)\right]} .
$$

Under squared error and Linex loss functions, we can obtain the Bayes estimator of the parameter $p$ by generating $\left(p^{j}, \alpha_{1}^{j}, \alpha_{2}^{j}\right) j=1,2, \cdots, M$ from the prior (34) and setting $u(\theta)=p$ in (23) and (24). The Bayes estimates of $\alpha_{1}$ and $\alpha_{2}$ can be similarly computed.

\section{Special cases}

\section{1) Upper order statistics}

If we put $m=0$ and $k=1$ in (35), $\left(\gamma_{r}=n-r+1\right)$, the posterior density function takes the form

$$
\pi^{*}(\theta \mid \boldsymbol{t}) \propto p_{1}^{b_{1}-1} p_{2}^{b_{2}-1} \exp \left[-\sum_{j=1}^{2} \frac{\left(\alpha_{j}-1\right)}{d_{j}}\right] \prod_{i=s}^{r}\left[p_{1} f_{1}\left(t_{i}\right)+p_{2} f_{2}\left(t_{i}\right)\right] \times\left[p_{1} R_{1}\left(t_{r}\right)+p_{2} R_{2}\left(t_{r}\right)\right]^{n-r} \sum_{\ell=0}^{s-1} \omega_{\ell}^{(s)}\left[p_{1} R_{1}\left(t_{s}\right)+p_{2} R_{2}\left(t_{s}\right)\right]^{\delta} .
$$

\section{2) Upper record values}

If we put $k=1$ in (36), $\left(\gamma_{r}=1\right)$, the posterior density function takes the form

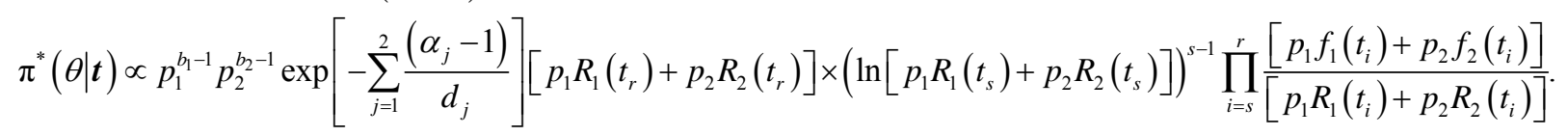

Under squared error and Linex loss functions, we can obtain the Bayes estimator of the parameter $p$, by generating $\left(p^{j}, \alpha_{1}^{j}, \alpha_{2}^{j}\right) j=1,2, \cdots, M$ from the prior (34) and setting $u(\theta)=p$ in (23) and (24). The Bayes estimates of $\alpha_{1}$ and $\alpha_{2}$ can be similarly computed.

\section{Simulation Study}

A comparison between ML and Bayes estimators, under either a squared error or a Linex loss functions, is made using a Monte Carlo simulation study in the two cases upper order statistics and upper record values according to the following steps:

1) For a given values of the prior parameters $\left(b_{1}, b_{2}\right)$ generate a random value $p$ from the $\operatorname{Beta}\left(b_{1}, b_{2}\right)$ distribution.

2) For a given values of the prior parameters $d_{j}$, for $j=1,2$, generate a random value $\alpha_{j}$ from the $\operatorname{LTE}\left(d_{j}\right)$ distribution.

3) Using the generated values of $p, \alpha_{1}$ and $\alpha_{2}$, we generate a random sample of size $n=(20,30,50)$ from a mixture of two $\operatorname{Gomp}\left(\alpha_{j}\right)$ components, $j=1,2$, as follows:

- generate two observations $u_{1}, u_{2}$ from $\operatorname{Uniform}(0,1)$.

- if $u_{1} \leq p$, then

$$
t=\frac{1}{\alpha_{1}} \log \left[1-\alpha_{1} \log \left(1-u_{2}\right)\right],
$$

otherwise

$$
t=\frac{1}{\alpha_{2}} \log \left[1-\alpha_{2} \log \left(1-u_{2}\right)\right] .
$$

- repeat above steps $n$ times to get a sample of size $n$.

4) The sample obtained in Step 3 is ordered.

5) The MLE's of the parameters $p, \alpha_{1}$ and $\alpha_{2}$ are obtained by solving the nonlinear Equations (31), by using Mathematica 6 .

6) Using the generated values of $p, \alpha_{1}$ and $\alpha_{2}$, we generate upper record values of size $n=(5,8,10)$ from a mixture of two $\operatorname{Gomp}\left(\alpha_{j}\right), j=1,2$ components.

7) The MLE's of the parameters $p, \alpha_{1}$ and $\alpha_{2}$ are obtained by solving the nonlinear Equations (33), by using Mathematica 6.

8) The Bayes estimates under squared error and Linex loss functions (BES, BEL), of $p, \alpha_{1}$ and $\alpha_{2}$ are computed, by using MCI forms (23) and (24), respectively.

9) The squared deviations $(\hat{\varepsilon}-\varepsilon)^{2}$ are computed for different samples and censoring sizes, where $\varepsilon$ stands for the parameter and $\hat{\varepsilon}$ its estimate (ML or Bayes).

10) The above Steps (3)-(9) are repeated 1000 times. The averages and the estimated risks (ER) are computed over the 1000 repetitions by averaging the estimates and the squared deviations, respectively.

The computational (our) results were computed by 
using Mathematica 6.0. In all above cases the prior parameters chosen as $b_{1}=4.5, b_{2}=3.2, d_{1}=1.2, d_{2}=2.3$, which yield the generated values of $p=0.524855$, $\alpha_{1}=1.47589$ and $\alpha_{2}=2.7255$ (as the true values). The true values of $R(t)$ and $H(t)$ when $t=0.5$, are computed to be $R(0.5)=0.414044$ and $H(0.5)=$
2.80864. The value of the shape parameter $a$ of the Linex loss function is $a=2$. The averages and the estimated risks (ER) are displayed in Tables 1-4. Figures 1 and 2 represent the estimated risks of the estimates in the case of upper order statistics. Figures 3 and 4 represent the estimated risks of the estimates

Table 1. (Upper order statistics) Averages and Estimated Risks (ER) of the estimates of $\theta=\left(p, \alpha_{1}, \alpha_{2}\right)$ for different samples and censoring sizes.

\begin{tabular}{|c|c|c|c|c|}
\hline$n(s, r)$ & Method & $\overline{\hat{p}} \quad E R(\overline{\hat{p}})$ & $\overline{\hat{\alpha}}_{1} \quad E R\left(\overline{\hat{\alpha}}_{1}\right)$ & $\overline{\hat{\alpha}}_{2} \quad E R\left(\overline{\hat{\alpha}}_{2}\right)$ \\
\hline \multirow{3}{*}{$20(2,14)$} & ML & $0.707005(0.19356)$ & $2.31845(1.46886)$ & $2.39962(0.850168)$ \\
\hline & $\mathrm{BL}$ & $0.616284(0.00864557)$ & $1.09815(0.14386)$ & $1.13638(2.52742)$ \\
\hline & BS & $0.643168(0.0141694)$ & $1.10888(0.136649)$ & $1.16379(2.44375)$ \\
\hline \multirow{6}{*}{$30(2,21)$} & ML & 0.714324 & 2.25684 & 2.32837 \\
\hline & & $(0.188562)$ & $(1.18964)$ & $(0.716555)$ \\
\hline & $\mathrm{BL}$ & 0.632902 & 1.022 & 1.12869 \\
\hline & & $(0.011701)$ & $(0.20618)$ & $(2.55009)$ \\
\hline & BS & 0.683938 & 1.02377 & 1.16001 \\
\hline & & $(0.0254091)$ & $(0.204632)$ & $(2.45121)$ \\
\hline \multirow{5}{*}{$\begin{array}{c}50 \\
(2,35)\end{array}$} & ML & 0.666667 & 2.17853 & 2.26631 \\
\hline & & $(0.181185)$ & $(0.806393)$ & $(0.516371)$ \\
\hline & & $(0.000574537)$ & $(0.20208)$ & $(2.74081)$ \\
\hline & $\mathrm{BS}$ & 0.552577 & 1.02767 & 1.07139 \\
\hline & & $(0.000797349)$ & $(0.200941)$ & $(2.73608)$ \\
\hline \multirow{3}{*}{$20(1,14)$} & ML & $0.710467(0.196735)$ & $2.36646(1.64159)$ & $2.44219(0.907496)$ \\
\hline & $\mathrm{BL}$ & $0.601193(0.005991)$ & $1.10452(0.138821)$ & $1.1209(2.57694)$ \\
\hline & BS & $0.623959(0.00994848)$ & $1.1156(0.131404)$ & $1.15205(2.48091)$ \\
\hline $\begin{array}{c}n \\
(s, r)\end{array}$ & Method & $\overline{\hat{p}} \quad E R(\overline{\hat{p}})$ & $\overline{\hat{\alpha}}_{1} \quad \operatorname{ER}\left(\overline{\hat{\alpha}}_{1}\right)$ & $\overline{\hat{\alpha}}_{2} \quad E R\left(\overline{\hat{\alpha}}_{2}\right)$ \\
\hline \multirow{4}{*}{$30(1,21)$} & MI & 0.725016 & 2.20851 & 2.28107 \\
\hline & $\mathrm{MIL}$ & $(0.195162)$ & $(1.03578)$ & $(0.692991)$ \\
\hline & $\mathrm{BL}$ & $0.607509(0.00766407)$ & $1.065(0.169001)$ & $1.10095(2.63928)$ \\
\hline & BS & $0.630063(0.0116613)$ & $1.06868(0.166023)$ & $1.10867(2.61438)$ \\
\hline \multirow{5}{*}{$50(1,35)$} & & $(0.183833)$ & $(0.758847)$ & $(0.522389)$ \\
\hline & $\mathrm{BL}$ & 0.458447 & 1.04342 & 1.05501 \\
\hline & & $(0.00464071)$ & $(0.187036)$ & $(2.79062)$ \\
\hline & BS & $0.464408(0.00402117)$ & $1.04364(0.186845)$ & $1.05637(2.7861)$ \\
\hline & ML & 0.695272 & 1.89954 & 2.38476 \\
\hline \multirow{4}{*}{$\begin{array}{c}20 \\
(1,20)\end{array}$} & & $(0.178062)$ & $(0.505669)$ & $(0.90054)$ \\
\hline & $\mathrm{BL}$ & 0.562142 & 1.62688 & 1.82389 \\
\hline & & $(0.00151982)$ & $(0.0668917)$ & $(0.85426)$ \\
\hline & $\mathrm{BS}$ & 0.589223 & 1.97985 & 2.83692 \\
\hline \multirow{5}{*}{$30(1,30)$} & & $(0.00429)$ & $(0.360228)$ & $(0.411247)$ \\
\hline & ML & $0.671677(0.1622)$ & $1.79666(0.353318)$ & $2.45167(1.18287)$ \\
\hline & BL & $0.556202(0.00130063)$ & $1.64213(0.0834779)$ & $1.83633(0.838901)$ \\
\hline & & 0.585439 & 1.96737 & 2.67792 \\
\hline & BS & $(0.004034)$ & $(0.352081)$ & $(0.375141)$ \\
\hline \multirow{3}{*}{$50(1,50)$} & ML & $0.626802(0.144386)$ & $1.70131(0.209794)$ & $2.49075(0.791618)$ \\
\hline & $\mathrm{BL}$ & $0.563246(0.00174849)$ & $1.651(0.0792463)$ & $1.8386(0.821578)$ \\
\hline & BS & $0.588489(0.00434187)$ & $1.93554(0.292391)$ & $2.65172(0.359827)$ \\
\hline
\end{tabular}


Table 2. (Upper order statistics) averages and estimated risks (ER) of the estimates of $R(t)$ and $H(t)$ for different sample and censoring sizes.

\begin{tabular}{|c|c|c|c|}
\hline$n(s, r)$ & Method & $\overline{\hat{R}}(t) E R(\overline{\hat{R}}(t))$ & $\overline{\hat{H}}(t) E R(\overline{\hat{H}}(t))$ \\
\hline \multirow{3}{*}{$20(2,14)$} & ML & $0.381661(0.00906794)$ & $3.68514(7.66423)$ \\
\hline & $\mathrm{BL}$ & $0.511856(0.00958999)$ & $1.74308(1.13692)$ \\
\hline & BS & $0.511923(0.00960227)$ & $1.7486(1.12574)$ \\
\hline \multirow{6}{*}{$30(2,21)$} & ML & 0.388581 & 3.43017 \\
\hline & & $(0.00706446)$ & $(2.94528)$ \\
\hline & BL & 0.51866 & 1.68457 \\
\hline & & $(0.0109467)$ & $(1.26369)$ \\
\hline & BS & 0.51867 & 1.6854 \\
\hline & & ( 0.0109488$)$ & $(1.26185)$ \\
\hline \multirow{6}{*}{$\begin{array}{c}50 \\
(2,35)\end{array}$} & ML & 0.396371 & 3.18718 \\
\hline & & $(0.00370546)$ & $(1.07172)$ \\
\hline & $\mathrm{BL}$ & 0.518651 & 1.68541 \\
\hline & & $(0.0109425)$ & $(1.26163)$ \\
\hline & BS & 0.518651 & 1.68546 \\
\hline & & $(0.0109426)$ & $(1.26153)$ \\
\hline \multirow{3}{*}{$20(1,14)$} & ML & $0.376971(0.0100604)$ & $3.85478(12.0168)$ \\
\hline & $\mathrm{BL}$ & $0.511715(0.00955799)$ & $1.74519(1.13219)$ \\
\hline & $\mathrm{BS}$ & $0.51177(0.00956825)$ & $1.7498(1.12281)$ \\
\hline$n(s, r)$ & Method & $\overline{\hat{R}}(t) \operatorname{ER}(\overline{\hat{R}}(t))$ & $\overline{\hat{H}}(t) E R(\overline{\hat{H}}(t))$ \\
\hline \multirow{3}{*}{$30(1,21)$} & ML & $0.393384(0.00610191)$ & 3.31504 \\
\hline & & & $(2.11435)$ \\
\hline & $\mathrm{BL}$ & $0.515905(0.0103771)$ & $1.70996(1.2072)$ \\
\hline \multirow{7}{*}{$50(1,35)$} & BS & $0.515913(0.0103787)$ & $1.71063(1.20575)$ \\
\hline & ML & 0.398975 & 3.14314 \\
\hline & & $(0.00348857)$ & $(0.93894)$ \\
\hline & $\mathrm{BL}$ & 0.518317 & 1.68844 \\
\hline & & $(0.0108729)$ & $(1.25485)$ \\
\hline & BS & $0.518318(0.0108731)$ & $1.68851(1.2547)$ \\
\hline & ML & 0.409763 & 2.962 \\
\hline \multirow{4}{*}{$\begin{array}{c}20 \\
(1,20)\end{array}$} & & $(0.00335753)$ & $(0.742865)$ \\
\hline & $\mathrm{BL}$ & 0.394839 & 2.58386 \\
\hline & & $(0.00226178)$ & $(0.154358)$ \\
\hline & BS & 0.398332 & 2.95451 \\
\hline \multirow{5}{*}{$30(1,30)$} & & $(0.0020448)$ & $(0.319495)$ \\
\hline & ML & $0.413582(0.00272424)$ & $2.881(0.51324)$ \\
\hline & $\mathrm{BL}$ & $0.398846(0.00209693)$ & $2.62951(0.174472)$ \\
\hline & & 0.401559 & 2.92977 \\
\hline & BS & $(0.00195636)$ & $(0.308405)$ \\
\hline \multirow{3}{*}{$50(1,50)$} & ML & $0.415782(0.00169979)$ & $2.82688(0.290495)$ \\
\hline & $\mathrm{BL}$ & $0.402934(0.00139306)$ & $2.67367(0.135707)$ \\
\hline & BS & $0.404751(0.001321)$ & $2.87568(0.186433)$ \\
\hline
\end{tabular}


Table 3. (Upper record values) Averages and Estimated Risks (ER) of the estimates of $\theta=\left(p, \alpha_{1}, \alpha_{2}\right)$ for different sample and censoring sizes.

\begin{tabular}{|c|c|c|c|c|}
\hline$n(s, r)$ & Method & $\overline{\hat{p}} \operatorname{ER}(\overline{\hat{p}})$ & $\overline{\hat{\alpha}}_{1} E R\left(\overline{\hat{\alpha}}_{1}\right)$ & $\overline{\hat{\alpha}}_{2} E R\left(\overline{\hat{\alpha}}_{2}\right)$ \\
\hline \multirow{3}{*}{$5(2,4)$} & ML & $0.631933(0.200897)$ & $2.67986(2.83816)$ & $2.89873(1.4118)$ \\
\hline & BL & $0.558404(0.00128567)$ & $1.74504(0.108009)$ & $1.98448(0.579141)$ \\
\hline & BS & $0.587338(0.00404486)$ & $2.28454(0.793391)$ & $3.46485(0.776266)$ \\
\hline \multirow{6}{*}{$8(2,7)$} & ML & 0.564415 & 2.61393 & 3.0953 \\
\hline & \multirow{3}{*}{$\mathrm{BL}$} & $(0.193903)$ & (2.11598) & $(5.09421)$ \\
\hline & & 0.545122 & 1.99517 & 2.21236 \\
\hline & & $(0.000661206)$ & $(0.36226)$ & $(0.334018)$ \\
\hline & \multirow{2}{*}{ BS } & 0.574543 & 2.59249 & 3.70339 \\
\hline & & $(0.00269061)$ & $(1.47151)$ & $(1.32978)$ \\
\hline \multirow{6}{*}{$\begin{array}{c}10 \\
(2,9)\end{array}$} & \multirow[t]{2}{*}{ ML } & 0.751296 & 2.14647 & 2.93067 \\
\hline & & $(0.19713)$ & $(0.632337)$ & (23.1693) \\
\hline & \multirow[t]{2}{*}{ BL } & 0.553808 & 1.86508 & 2.11734 \\
\hline & & $(0.000924086)$ & $(0.227293)$ & $(0.431788)$ \\
\hline & \multirow[t]{2}{*}{ BS } & 0.580802 & 2.30151 & 3.30646 \\
\hline & & $(0.00320725)$ & $(0.800718)$ & $(0.509574)$ \\
\hline \multirow{3}{*}{$5(1,4)$} & ML & $0.626796(0.2019)$ & $2.62149(2.54316)$ & $2.85242(1.32977)$ \\
\hline & $\mathrm{BL}$ & $0.561015(0.00133488)$ & $1.74589(0.104393)$ & $1.92257(0.667007)$ \\
\hline & BS & 0.5882 & 2.27422 & 3.24856 \\
\hline$n(s, r)$ & Method & $\overline{\hat{p}} \quad E R(\overline{\hat{p}})$ & $\overline{\hat{\alpha}}_{1} \quad E R\left(\overline{\hat{\alpha}}_{1}\right)$ & $\overline{\hat{\alpha}}_{2} \quad E R\left(\overline{\hat{\alpha}}_{2}\right)$ \\
\hline \multirow{4}{*}{$8(1,7)$} & \multirow{2}{*}{ ML } & 0.553391 & 2.58457 & 2.9875 \\
\hline & & $(0.194865)$ & $(1.69481)$ & $(2.2188)$ \\
\hline & BL & $0.553064(0.00100325)$ & $2.00503(0.360188)$ & $2.14377(0.404423)$ \\
\hline & $\mathrm{BS}$ & $0.582772(0.0035541)$ & $2.56838(1.36471)$ & $3.76664(1.4979)$ \\
\hline \multirow{6}{*}{$10(1,9)$} & \multirow[t]{2}{*}{ ML } & 0.737692 & 2.12728 & 2.69955 \\
\hline & & $(0.195127)$ & $(0.627068)$ & $(5.2213)$ \\
\hline & \multirow[t]{2}{*}{$\mathrm{BL}$} & 0.551621 & 1.86627 & 2.08316 \\
\hline & & $(0.000954124)$ & $(0.218747)$ & $(0.468689)$ \\
\hline & BS & $0.583116(0.00359548)$ & $2.24422(0.690718)$ & $3.34005(0.632348)$ \\
\hline & \multirow[t]{2}{*}{ ML } & 0.69058 & 2.25104 & 2.6748 \\
\hline \multirow{4}{*}{$\begin{array}{c}5 \\
(1,5)\end{array}$} & & $(0.195468)$ & $(1.41082)$ & $(1.11396)$ \\
\hline & \multirow[t]{2}{*}{ BL } & 0.555479 & 1.72972 & 1.91339 \\
\hline & & $(0.00102412)$ & $(0.116361)$ & $(0.699269)$ \\
\hline & \multirow[t]{2}{*}{ BS } & 0.584415 & 2.21588 & 3.26564 \\
\hline \multirow{5}{*}{$8(1,8)$} & & $(0.00363311)$ & $(0.7209)$ & $(0.576103)$ \\
\hline & ML & $0.671123(0.196826)$ & $2.28674(1.0187)$ & $2.85737(4.12699)$ \\
\hline & \multirow[t]{2}{*}{ BL } & $0.538713(0.000392836)$ & $1.89649(0.283578)$ & $2.10966(0.470518)$ \\
\hline & & 0.56659 & 2.4252 & 3.41334 \\
\hline & BS & $(0.00190609)$ & $(1.13393)$ & $(0.749679)$ \\
\hline \multirow{3}{*}{$\begin{array}{c}10 \\
(1,10)\end{array}$} & ML & $0.789522(0.194147)$ & $2.00076(0.478516)$ & $2.67385(1.92451)$ \\
\hline & $\mathrm{BL}$ & $0.547329(0.000585634)$ & $1.82498(0.181567)$ & $2.03864(0.53141)$ \\
\hline & BS & $0.575719(0.00265766)$ & $2.23599(0.660433)$ & $3.23598(0.476606)$ \\
\hline
\end{tabular}


Table 4. (Upper record values) Averages and Estimated Risks (ER) of the estimates of $R(t)$ and $H(t)$ for different sample and censoring sizes.

\begin{tabular}{|c|c|c|c|}
\hline$n(s, r)$ & Method & $\overline{\hat{R}}(t) E R(\overline{\hat{R}}(t))$ & $\overline{\hat{H}}(t) \operatorname{ER}(\overline{\hat{H}}(t))$ \\
\hline \multirow{3}{*}{$5(2,4)$} & ML & $0.342028(0.0172756)$ & $5.66515(237.48)$ \\
\hline & $\mathrm{BL}$ & $0.344783(0.00674596)$ & $2.68139(0.0733731)$ \\
\hline & BS & $0.353593(0.00543755)$ & $3.59821(1.31366)$ \\
\hline \multirow{6}{*}{$8(2,7)$} & ML & 0.335959 & 4.4366 \\
\hline & & $(0.0132904)$ & $(10.0859)$ \\
\hline & BL & 0.312673 & 3.05881 \\
\hline & & $(0.0125119)$ & $(0.196192)$ \\
\hline & BS & 0.320432 & 4.07878 \\
\hline & & $(0.0109407)$ & $(2.75144)$ \\
\hline \multirow{6}{*}{$\begin{array}{c}10 \\
(2,9)\end{array}$} & ML & 0.392196 & 3.26384 \\
\hline & & $(0.00425724)$ & $(1.69679)$ \\
\hline & $\mathrm{BL}$ & 0.349632 & 2.86293 \\
\hline & & $(0.00541351)$ & $(0.118538)$ \\
\hline & BS & 0.355924 & 3.46669 \\
\hline & & $(0.00461905)$ & $(0.874738)$ \\
\hline \multirow{3}{*}{$5(1,4)$} & ML & $0.348583(0.0161086)$ & $4.99658(64.6011)$ \\
\hline & BL & $0.352821(0.00530487)$ & $2.69339(0.0650445)$ \\
\hline & BS & $0.360458(0.00428379)$ & $3.5342(1.30239)$ \\
\hline$n(s, r)$ & Method & $\overline{\hat{R}}(t) E R(\overline{\hat{R}}(t))$ & $\overline{\hat{H}}(t) E R(\overline{\hat{H}}(t))$ \\
\hline \multirow{4}{*}{$8(1,7)$} & MI & $0338792(0012543)$ & 4.31724 \\
\hline & & $0.338 / 92(0.012343)$ & $(6.94347)$ \\
\hline & BL & $0.31614(0.0115378)$ & $2.99744(0.157374)$ \\
\hline & $\mathrm{BS}$ & $0.323935(0.0100044)$ & $3.95132(2.20381)$ \\
\hline \multirow{6}{*}{$10(1,9)$} & ML & 0.394473 & 3.19236 \\
\hline & & $(0.00356277)$ & $(1.10688)$ \\
\hline & $\mathrm{BL}$ & 0.350405 & 2.87756 \\
\hline & & $(0.00522127)$ & $(0.100219)$ \\
\hline & BS & $0.356443(0.00444454)$ & $3.48105(0.768389)$ \\
\hline & ML & 0.379553 & 3.97702 \\
\hline \multirow{4}{*}{$\begin{array}{c}5 \\
(1,5)\end{array}$} & & $(0.0101978)$ & $(60.4934)$ \\
\hline & $\mathrm{BL}$ & 0.356758 & 2.66213 \\
\hline & & $(0.00535798)$ & $(0.0984434)$ \\
\hline & BS & 0.364529 & 3.41487 \\
\hline \multirow{5}{*}{$8(1,8)$} & & $(0.00435279)$ & $(1.22003)$ \\
\hline & ML & $0.372725(0.0076614)$ & $3.64809(3.4103)$ \\
\hline & $\mathrm{BL}$ & $0.331679(0.00880409)$ & $2.93711(0.172037)$ \\
\hline & & 0.338926 & 3.74408 \\
\hline & BS & $(0.0076069)$ & $(1.62913)$ \\
\hline \multirow{3}{*}{$10(1,10)$} & ML & $0.406745(0.00339404)$ & $3.01535(1.01302)$ \\
\hline & $\mathrm{BL}$ & $0.353242(0.004766)$ & $2.84017(0.097151)$ \\
\hline & BS & $0.359484(0.0040121)$ & $3.38218(0.568505)$ \\
\hline
\end{tabular}



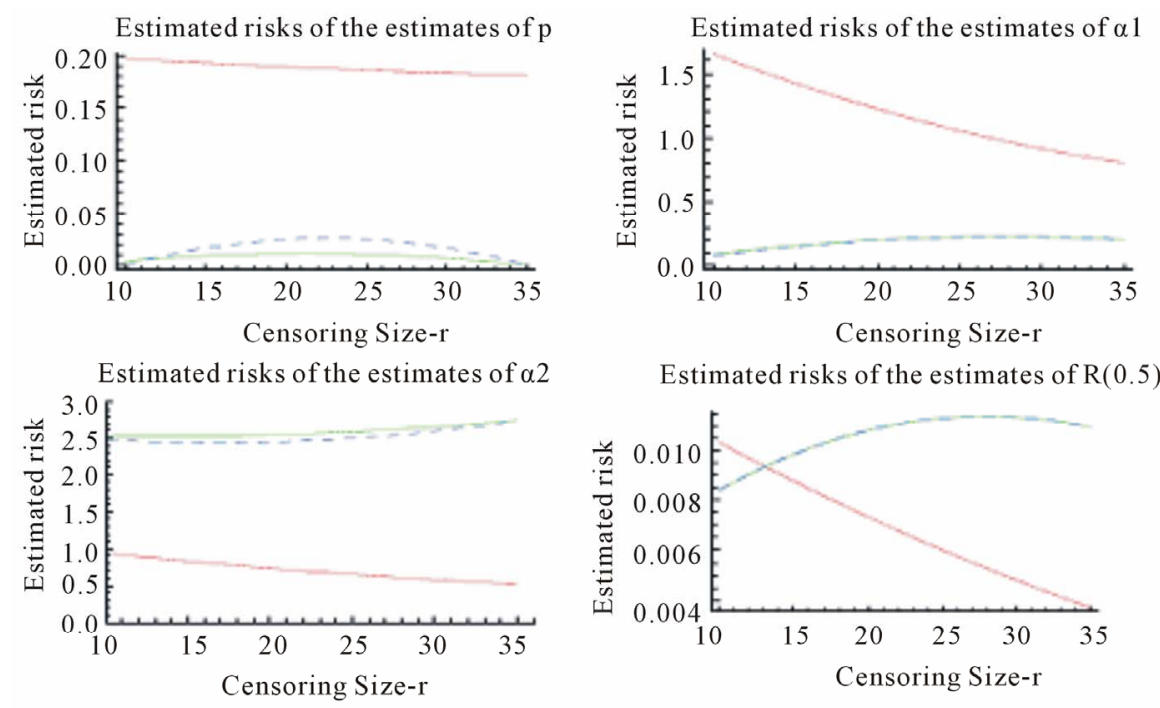

Estimated risks of the estimates of $\mathrm{R}(0.5)$
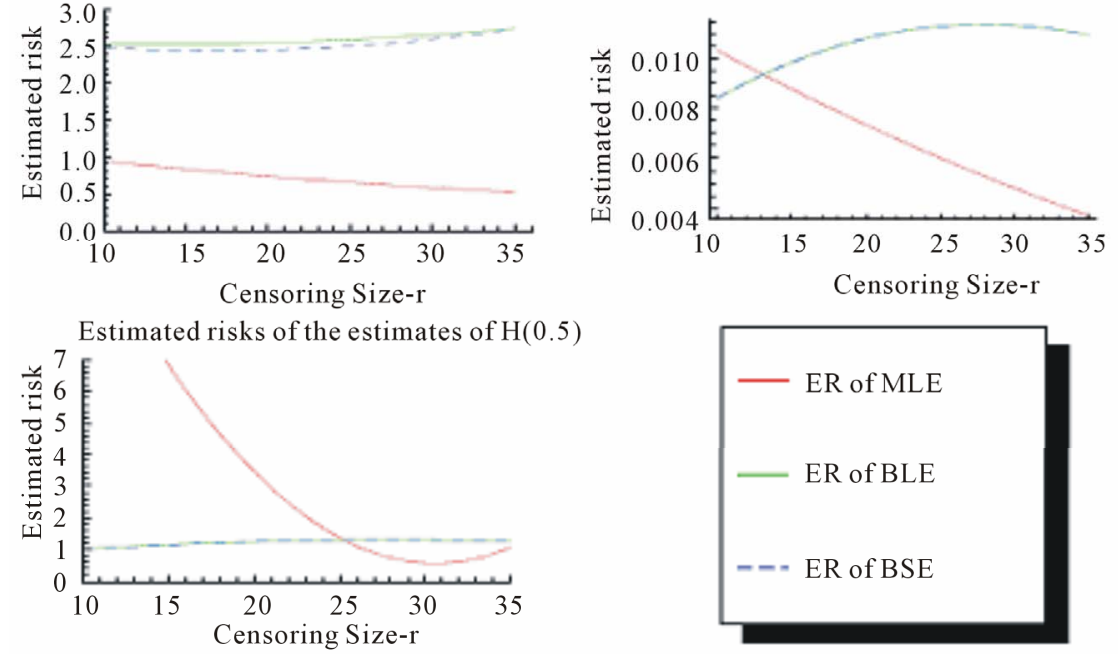

Figure 1. Estimated Risks (ER) of the estimates of $\theta=\left(p, \alpha_{1}, \alpha_{2}\right), R(t)$ and $H(t)$ based on doubly Type II censored samples $(s=2)$.
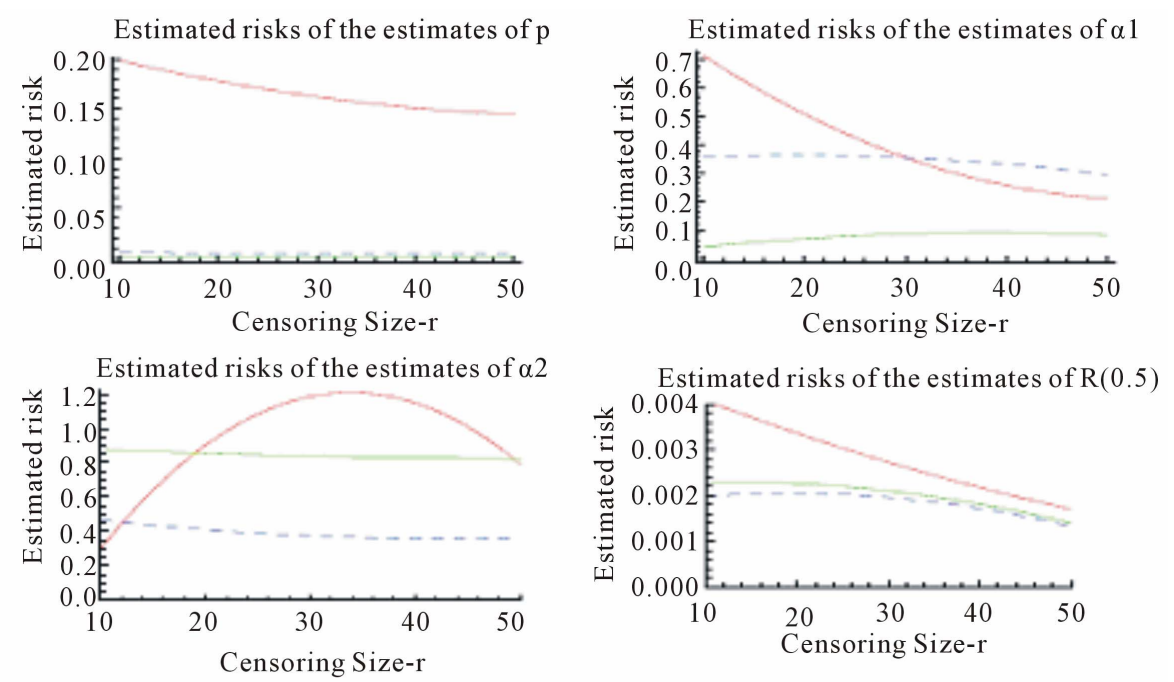

Estimated risks of the estimates of $\mathrm{H}(0.5)$
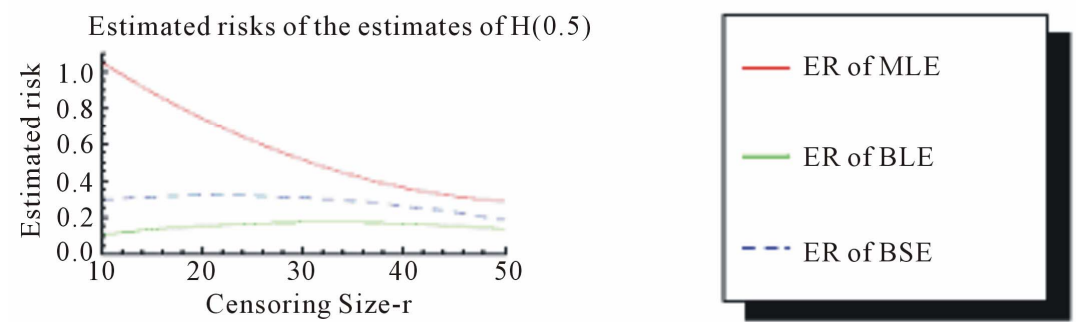

Figure 2. Estimated Risks (ER) of the estimates of $\theta=\left(p, \alpha_{1}, \alpha_{2}\right), R(t)$ and $H(t)$ complete samples. 


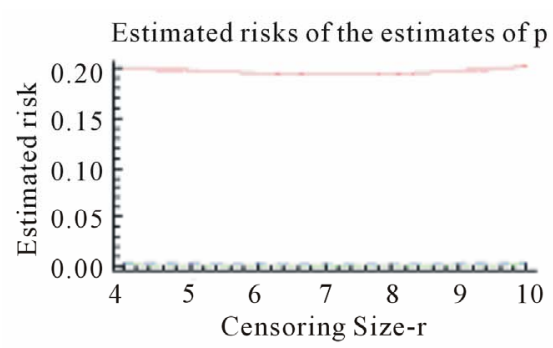

Estimated risks of the estimates of $\alpha 2$

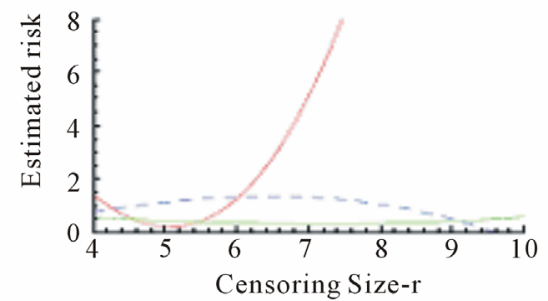

Estimated risks of the estimates of $\mathrm{H}(0.5)$

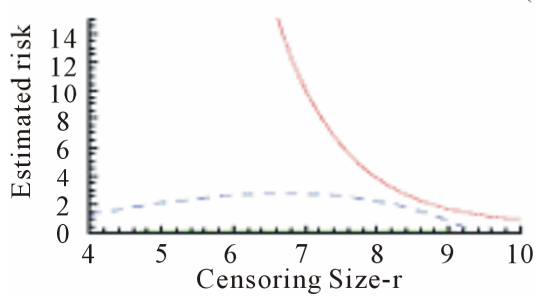

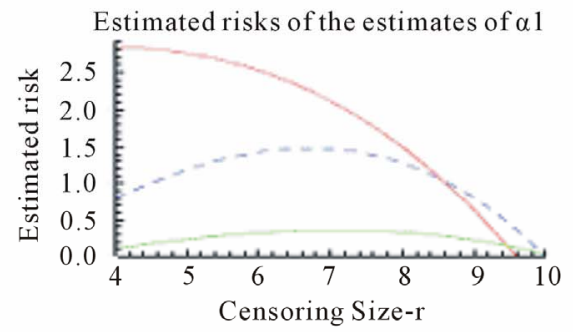

Estimated risks of the estimates of $\mathrm{R}(0.5)$
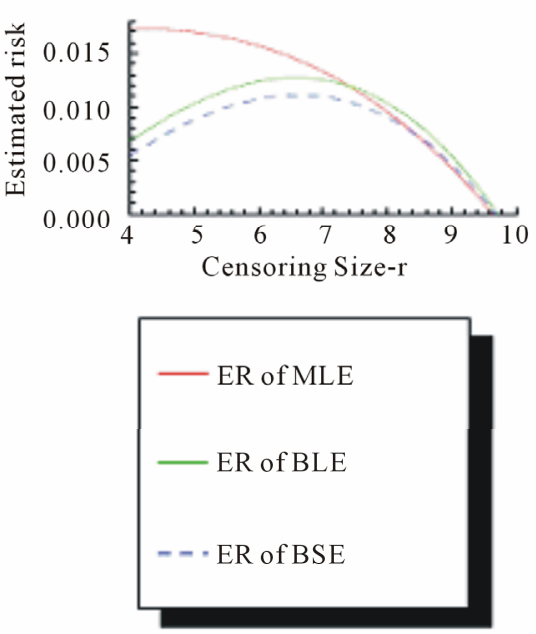

Figure 3. Estimated Risks (ER) of the estimates of $\theta=\left(p, \alpha_{1}, \alpha_{2}\right), R(t)$ and $H(t)$ based on doubly Type II censored samples $(s=2)$.

Estimated risks of the estimates of $\mathrm{p}$

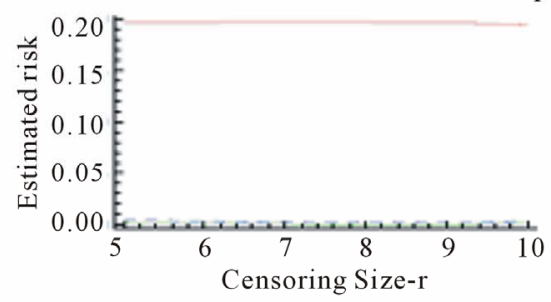

Estimated risks of the estimates of $\alpha 2$

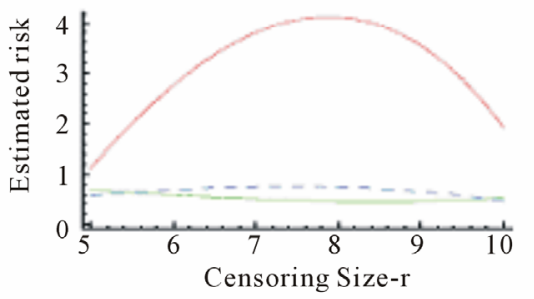

Estimated risks of the estimates of $\mathrm{H}(0.5)$

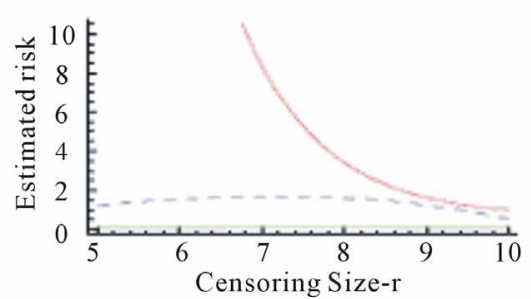

Estimated risks of the estimates of $\alpha 1$

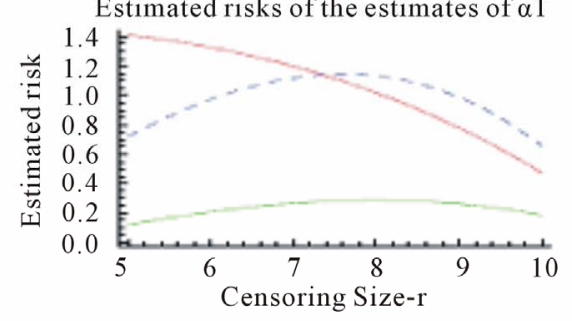

Estimated risks of the estimates of $\mathrm{R}(0.5)$
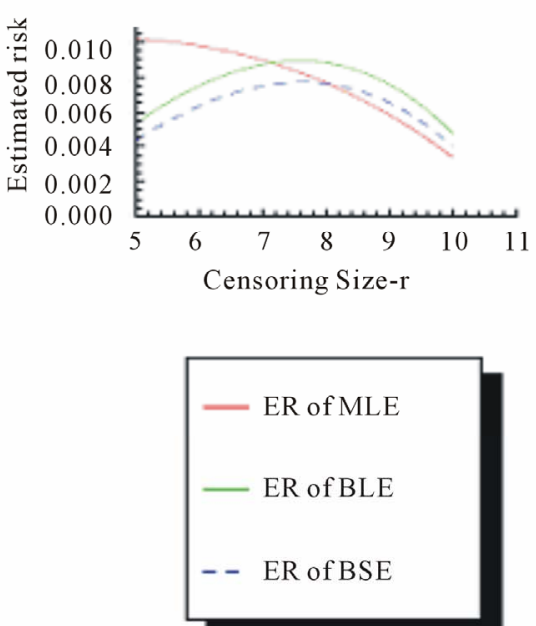

Figure 4. Estimated Risks (ER) of the estimates of $\theta=\left(p, \alpha_{1}, \alpha_{2}\right), R(t)$ and $H(t)$ complete samples. 
in the case of upper record values.

\section{Concluding Remarks}

1) Estimation of the parameters of the finite mixture model of two Gompertz distributions are considered from a Bayesian approach based on gos's. A compareson between ML and Bayes estimators, under either a squared error loss or a Linex loss, is made by using a Monte Carlo simulation study in both two cases considering order statistics and upper record values cases.

2) From Tables 1 and 2, we see that in most of the considered cases, the ER's of the estimates decrease as $n$ increases. In complete sample case, the Bayes estimates of $p, \alpha_{1}$ and HRF under Linex loss function have the smallest ER's as compared with their corresponding estimates under squared error loss function or MLE', while the ER's of the Bayes estimates of $\alpha_{2}$ and $R F$ under squared error loss functions are the smallest estimated risks. For censored samples, the Bayes estimates of $p$ under Linex loss function have the smallest ER's as compared with their corresponding estimates under squared error loss function or MLE's. While, the Bayes estimates (against the proposed prior) of $\alpha_{1}$ and HRF under squared error loss function have the smallest ER's as compared with their corresponding estimates. It is observed that MLE's for $H R F$ perform best when sample size $n$ is increased. Also, we note that the MLE's of $\alpha_{2}$ and $R F$ have the smallest ER's as compared with Bayes estimates.

3) From Tables $\mathbf{3}$ and 4, we see that the Bayes estimates (against the proposed prior) of the parameters and HRF under Linex loss function have the smallest ER's as compared with their corresponding estimates under squared error loss function or MLE's. While, the Bayes estimates of $\alpha_{2}$ (for complete sample) and $R F$ under squared error loss function have the smallest ER's as compared with both Bayes estimates under Linex loss function or the MLE's. Also, it is observed that MLE's for RF perform best when sample size $n$ is increased.

4) If the mixing proportion $p$ is known, [2] estimated the parameters $\alpha_{1}, \alpha_{2}$, reliability and hazard rate functions based on Types I and II censored samples.

\section{REFERENCES}

[1] A. S. Papadapoulos and W. J. Padgett, "On Bayes Estimation for Mixtures of Two Exponential-Life-Distributions from Right-Censored Samples," IEEE Transactions on Reliability, Vol. 35, No. 1, 1986, pp. 102-105. doi:10.1109/TR.1986.4335364

[2] E. K. AL-Hussaini, G. R. Al-Dayian and S. A. Adham, "On Finite Mixture of Two-Component Gompertz Lifetime Model," Journal of Statistical Computation and Simulation, Vol. 67, No. 1, 2000, pp. 1-20.
[3] E. K. AL-Hussaini and S. F. Ateya, "Bayes Estimations under a Mixture of Truncated Type I Generalized Logistic Components Model," Journal of Applied Statistical Science, Vol. 4 No. 2, 2005, pp. 183-208.

[4] Z. F. Jaheen, "On Record Statistics from a Mixture of Two Exponential Distributions," Journal of Statistical Computation and Simulation, Vol. 75, No. 1, 2005, pp. 111. doi:10.1080/00949650410001646924

[5] E. K. AL-Hussaini and R. A. AL-Jarallah, "Bayes Inference under a Finite Mixture of Two Compound Gompertz Components Model," Cairo University, 2006.

[6] K. S. Sultan, M. A. Ismail and A. S. Al-Moisheer, "Mixture of Two Inverse Weibull Distributions: Properties and Estimation," Computational Statistics \& Data Analysis, Vol. 51, No. 11, 2007, pp. 5377-5387.

doi:10.1016/j.csda.2006.09.016

[7] U. Kamps, "A Concept of Generalized Order Statistics," Journal of Statistical Planning and Inference, Vol. 48, No. 1, 1995, pp. 1-23. doi:10.1016/0378-3758(94)00147-N

[8] M. Ahsanullah, "Generalized Order Statistics from Two Parameter Uniform Distribution," Communications in Statistics-Theory and Methods, Vol. 25, No. 10, 1996, pp. 2311-2318. doi:10.1080/03610929608831840

[9] U. Kamps and U. Gather, "Characteristic Property of Generalized Order Statistics for Exponential Distributions," Applied Mathematics (Warsaw), Vol. 24, No. 4, 1997, pp. 383-391.

[10] E. Cramer and U. Kamps, "Relations for Expectations of Functions of Generalized Order Statistics," Journal of Statistical Planning and Inference, Vol. 89, No. 1-2, 2000, pp. 79-89. doi:10.1016/S0378-3758(00)00074-4

[11] M. Habibullah and M. Ahsanullah, "Estimation of Parameters of a Pareto Distribution by Generalized Order Statistics," Communications in Statistics-Theory and Methods, Vol. 29, No. 7, 2000, pp. 1597-1609. doi:10.1080/03610920008832567

[12] E. K. AL-Hussaini and A. A. Ahmad, "On Bayesian Predictive Distributions of Generalized Order Statistics," Metrika, Vol. 57, No. 2, 2003, pp. 165-176. doi:10.1007/s001840200207

[13] E. K. AL-Hussaini, "Generalized Order Statistics: Prospective and Applications," Journal of Applied Statistical Science, Vol. 13, No. 1, 2004, pp. 59-85.

[14] Z. F. Jaheen, "On Bayesian Prediction of Generalized Order Statistics," Journal of Applied Statistical Science, Vol. 1, No. 3, 2002, pp.191-204.

[15] Z. F. Jaheen, "Estimation Based on Generalized Order Statistics from the Burr Model," Communications in Statistics-Theory and Methods, Vol. 34, No. 4, 2005, pp. 785-794. doi:10.1081/STA-200054408

[16] A. A. Ahmad, "Relations for Single and Product Moments of Generalized Order Statistics from Doubly Truncated Burr Type XII Distribution," Journal of the Egyptian Mathematical Society, Vol. 15, No. , 2007, pp. 117128.

[17] A. A. Ahmad, "Single and product Moments of Generalized order Statistics from Linear Exponential Distribution," Communications in Statistics-Theory and Meth- 
ods, Vol. 37, No. 8, 2008, pp. 1162- 1172. doi:10.1080/03610920701713344

[18] S. F. Ateya and A. A. Ahmad, "Inferences Based on Generalized Order Statistics under Truncated Type I generalized Logistic Distribution," Statistics, Vol. 45, No. 4, 2011, pp. 389-402. doi:10.1080/02331881003650149

[19] T. A. Abu-Shal and A. M. AL-Zaydi, "Estimation Based on Generalized Order Statistics from a Mixture of Two Rayleigh Distributions," International Journal of Statistics and Probability, Vol. 1, No. 2, 2012, pp. 79-90.

[20] T. A. Abu-Shal and A. M. AL-Zaydi, "Prediction Based on Generalized Order Statistics from a Mixture of Rayleigh Distributions Using MCMC Algorithm," Open Journal of Statistics, Vol. 2, No. 3, 2012, pp. 356-367. doi:10.4236/ojs.2012.23044

[21] B. S. Everitt and D. J. Hand, "Finite Mixture Distributions," University Press, Cambridge, 1981. doi:10.1007/978-94-009-5897-5

[22] D. M. Titterington, A. F. M. Smith and U. E. Makov, "Statistical Analysis of Finite Mixture Distributions," John Wiley and Sons, New York, 1985.

[23] G. J. McLachlan and K. E. Basford, "Mixture Models: Inferences and Applications to Clustering," Marcel Dekker, New York, 1988.

[24] H. Teicher, "Identifiability of Finite Mixtures," The Annals of Mathematical Statistics, Vol. 34, No. 4, 1963, pp.
1265-1269. doi:10.1214/aoms/1177703862

[25] E. K. AL-Hussaini and K. E. Ahmad, "On the Identifiability of Finite Mixtures of Distributions," Transactions on Information Theory, Vol. 27, No. 5, 1981, pp. 664668.

[26] K. E. Ahmad, "Identifiability of Finite Mixtures Using a New Transform," The Annals of Mathematical Statistics, Vol. 40, No. 2, 1988, pp. 261-265. doi:10.1007/BF00052342

[27] A. A. Ahmad, "On Bayesian Interval Prediction of Future Generalized Order Statistics Using Doubly Censoring," Statistics, Vol. 45, No. 5, 2011, pp. 413- 425. doi: $10.1080 / 02331881003650123$

[28] U. Kamps, "A Concept of Generalized Order Statistics," Journal of Statistical Planning and Inference, Vol. 48, No. 1, 1995, pp. 1-23. doi:10.1016/0378-3758(94)00147-N

[29] E. K. AL-Hussaini, "Prediction, Observables from a General Class of Distributions," Journal of Statistical Planning and Inference, Vol. 79, No. 1, 1999, pp. 79-91. doi:10.1016/S0378-3758(98)00228-6

[30] S. J. Press, "Subjective and Objective Bayesian Statistics: Principles, Models and Applications," Wiley, New York, 2003. 